\title{
OBSERVATIONS ON THE THERAPEUTIC VIRTUE OF THE PURIFIED VACCINIA LYMPH ON BRONCHIAL ASTHMA*
}

\author{
By HIDETAKe . YAOI \\ National Institute of Health of Japan, Tokyo
}

It has already been reported by the author that the production of antibodies is distinctly accelerated when a bacterial vaccine is applied in combination with the purified vaccinia lymph.

Furthermore, a prompt suppression of paroxysms especially in the case of whooping cough, which can never be explained by the said rapid formation of antibodies alone, has led us to assume the existence of antiallergic action inherent to the living vaccinia virus.

An experiment to ascertain such influence proved a marked desensitizing action by the anaphylactic shock test upon guinea pigs.

Namely, when guinea pigs, sensitized to horse serum, were treated with a small amount. of purified vaccinia virus prior to the provocative dose of horse serum, proved to be exempt from the anaphylactic shock. Purified vaccinia lymph inactivated by heating showed no 'such effect ${ }^{1-8}$ ).

The author has also observed that Vitamin $B_{2}$ shows antianaphylactic efficacy but not as active as the purified vaccinia $1 \mathrm{pmph}$, but superior to Vitamin $\mathrm{C}^{9}$ ).

These findings have led us to examine the effect of purified vaccinia virus on bronchial asthma.

On 85 patients who have been treated by the author in Tokyo since 1942 will be described at this time.

Of this group, one was a case of cardiac asthma, and 3 were the cases of so-called asthmatic tuberculosis. The age of the treated patients varied from 2 to 66 years old.

Among these cases 34 had anamnesis' of so-called infantile asthma, and 11 others were suffering from at the time of treatment. Congenital predisposition to asthma was perceived in 11 cases, the fact which suggests that there may have been a still greater number of similar instances.

Disposition or causes of the seizure considered were exudative constitution, autopoisoning, eczema, nasal catarrh and angina, also inhalation of gases (of charcoal, poisonous, pyrethrinic, etc.), post-operational, exhausting labour, colds, bronchitis, measles, whooping cough and pneumonia. Change of climate, rain or shine, humidity, chill, topographical and housing defect, malnutrition and especially excessive eating.

Mental and physical exhaustion, menstruation in women, pregnancy, influence of specific allergen, etc., have also been regarded as the possible direct factors.

Seasonal influence seemed to be of special importance; spring and autumn, when a

* First published in Japanese (H. Yaoi : Therapeutic experiments of bronchial asthma by means of purified vaccinia lymph, Kiso to Rinsho, Vol. 1, No. 1. 25-32, 1947) 
climatic change takes place, particularly during the months of September to November, are conspicuous. However, some may develop paroxysms at any time of the year, while in others it is brought on during the cooler months only or in the summer exclusively.

The duration of the disease was from several to thirty years, and, the effectiveness of the purified vaccinia lymph as to control asthmatic seizure seems unrelated to the history of the disease, including previous treatments.

\section{EXPERIMENTAL}

\section{Injection . Technic :}

The doses in adults (revaccinated) are as follows :

The first injection of $1.0 \mathrm{cc}$ and $2.0 \mathrm{cc}$ after 48 hours, of the purified vaccinia lymph subcutaneously; 2 or $3 \mathrm{cc}$ are injected during 2 or 3 days following close observations thus completing one course.

Further applications may be practiced if deemed necessary.

A few hours after the first dose, many patients expressed some improvement, 'feeling warmer and looking better, etc. But in very rare cases the whoop seemed to be rather provoked by the injection. Prophylactic application of the remedy seemed of no avail.

But, when any sign or premise of the attack is shown a dose or two would usually satisfactorily check an outbreak.

A larger dose of $5.0 \mathrm{cc}$ has shown to be of no special merit although quite harmless, while smaller doses of $0.3-0.5 \mathrm{cc}$ have often been found quite sufficient. The latter fact was well observed by the author when he applied it among the natives in Java during the year 1944 .

The author has always refrained from applying the new remedy in patients who were under any other treatments, in order to be sure that the result observed was wholly due to the effect of the present method of treatment.

\section{Symptoms after Injection:}

Prompt improvement of complexion, warm and comfortable feelings, easier pulsation of the heart, refreshing and pleasant sensation; good rest and sleep, especially the warmth in feet, are expressed by, many patients.

But in very rare cases, namely, in three among 85 cases the asthmatic attack seemed to be rather provoked by the injection, and there were other cases in which the local allergic reaction due to vaccinia virus was markedly elicited, or a rise of temperature noted, although those extraordinary ill-effects were all' of transitory nature and lasted but for a short while.

\section{Effectiveness of the Injection:}

Results of application of the present method of treatment in said 85 cases may be classified into three groups: 
(1) Proved most effective, including complete cure in 55 cases or $64.7 \%$

(2) Effective to a certain extent in 12 cases or $14.1 \%$

(3) Proving no particular effectiveness in 18 cases or $21.2 \%$

\section{Total : 85 cases $100.0 \%$}

Thus, many cases were markedly improved by the present method of treatment and remained relatively well for as long as several months or even a year. Furthermore, in the majority of cases, improvement as judged by a decrease of severity or frequency of attacks was detected.

Among those who seemed uninfluenced by the new remedy, 11 cases or $13 \%$ were understood to have really received no benefit, while others were those who have given up further treatment after. single (one case), two (5 cases) or three (one case) injections.

Most effective results were seen among aged cases followed by infants, as in the following table :

\begin{tabular}{|c|c|c|c|c|}
\hline Groups & Cases & Most effective & Effective & Uninfluenced \\
\hline Infants $\quad(2-12)$ & 22 & $14(63.6 \%)$ & $4 .(18.2 \%)$ & $4 \quad(18.2 \%)$ \\
\hline Adults $\quad(14-49)$ & 48 & $27(56.2 \%)$ & $7(14.7 \%)$ & $14 \quad(29.1 \%)$ \\
\hline Advanced $(50-66)$ & 15 & $14(93.3 \%)$ & $1(6.6 \%)$ & Nil. \\
\hline Total & 85 & $55(64.7 \%)$ & $12 \cdot(14 \%)$ & $18 \cdot(21.3 \%)$ \\
\hline
\end{tabular}

The author has also tried the haemolytic streptococcic toxoid in combination with the purified vaccinia lymph and has witnessed especially remarkable effects on cases with sore throat.

\section{EXAmple of CAses :}

Case 12:- Male, age 12. Admitted on June 3, 1943.

Exudative constitution with pronounced predisposition to asthma (mother and three brothers being reported to have developed asthmatic attacks after measleș). The first seizure was brought on after having suffered from measles and pneumonia at 7 years of age, which have since exhibited a lasting effect on the rest of his life. Tonsils tremendously swollen.

Six injections were given:

1st injection, $0.5 \mathrm{cc}$ of the lymph with $1.0 \mathrm{cc}$ of toxoid 2nd injection, $0.5 \mathrm{cc}$ of the $1 \mathrm{ymph}$ with $2.0 \mathrm{cc}$ of toxoid 3rd injection, (without the lymph) $3.0 \mathrm{cc}$ of toxoid. 4th injection, (without the lymph) $3.0 \mathrm{cc}$ of toxoid 5 th injection, (without the lymph) $3.0 \mathrm{cc}$ of toxoid 6 th injection, (without the lymph) $3.0 \mathrm{cc}$ of toxoid

The first and the second injections showed but little reaction, and the third (haemolytic streptococcic toxoid alone) induced an asthmatic attack 30 minutes after the injection, 
the fourth and fifth doses also caused similar effects but the seizures were found to become less severer each time and entirely disappeared after the sixth injection. Tonsillar swelling diminished after the third injection.

In next example the purified vaccinia lymph was applied in combination with Vitamin $\mathbf{B}_{2}$.

Case 1:-Male adult, age 40. Admitted on Sep. 1, 1942.

The first asthmatic seizure was experienced during the autumn 1927, and suffererd from attacks ever since between September and November, year after year. Symptoms were said to have been so severe that no remedy of known reputation has ever done ary good.

Treatment started in the early in September, 1942.

First with two injections a week of $1.0 \mathrm{cc}$ of Bioflavin (Vitamin $\mathrm{B}_{2}$ ), and the purified vaccinia lymph was tried only when a sign of the asthmatic fit was detected. Results were remarkably favorable and no seizure has been breaking out since that year.

There is another case (a lady, 50) who was treated solely with Vitamin $B_{2}$ with a satisfactory result.

I. M., Vitamin $B_{1}$, and Vitamin $C$ have also been tried in combination with the purified vaccinia lymph but without benefit.

Five miscellaneous cases:

There were three cases of tuberculosis among 85 dealt with in this report. Two of these three eventually died and the last one is still alive. Another died after developing acute lobar pneumonia and the other from cardiac asthma. Four deaths have thus been registered out of 85 , or $4.7 \%$ mortality. It is noteworthy, however, that in all these cases there was a marked improvement in asthma, as noted in the following lines:-

(1) Case 6:-Male, age 37. Admitted on April 10, 1943.

Old spells of asthma for five years with severe cough, expectoration and dyspnoea.

Symptoms disappeared after two injections of the purified vaccinia lymph and suffered no longer from asthmatic attack until he died of advanced pulmonary tuberculosis on August 14th, the next year.

(2) Case 57 :- Male, age 39.

Suffered from asthma since childhood which grew severer and severer after his teens. Ephedrine, adrenalin, etc., were tried in vain.

Eight injections of the purified vaccinia lymph were made in four months' period between December 30th and the end of April of the following year, resulting in almost complete cessation of the symptoms. Though suffered no longer from asthmatic attack, he died rather suddenly of pulmonary tuberculosis early in June.

(3) Case 58 :- Male, age 65. Admitted on April 15th, 1944.

Asthmatic symptoms induced by, "colds", apparently of tuberculous character, some five years previously.

The first injection of $0.5 \mathrm{cc}$ on April 19th, proved satisfactory, and the second of 0.8 cc was made on July 7th, which suppressed the outbreak till the end of September when a mild sign was seen. The third injection of $0.4 \mathrm{cc}$ on October 6 th suppressed the disease for good one year till the symptoms in moderate degree with fever of $39.0^{\circ} \mathrm{C}$ sent him 
to bed for a week, while insomnia, orthophnoea, etc., disturbed him. The fourth injection of $4.0 \mathrm{cc}$ made on December 11th, 1945, seemed to have cured the malady almost completely. Tubercle bacilli were still proved positive in sputum in January 1946.

(4) Case $10:-$ Male, age 29. Admitted on May 15th, 1943.

Obstinate bronchial asthma for two solid years, cough; expectorations, dispnoea and palpitations all the year round.

Three injections of $0.5,2.0$ and $2.0 \mathrm{cc}$ with a week's interval induced a favorable turn and the fourth made on June 8 th with $5.0 \mathrm{cc}$ effected a permanent cure.

Acute lobar pneumonia developed after years will be of no concern to his asthmatic history.

(5) Case 13:- Male, age 50. Admitted on June 1st, 1943.

Cardiac asthma for five years and bronchial signs with vagotony in later days. Being confined to bed all the while.

Two injections of 1.0 and $2.0 \mathrm{cc}$ with a day's interval produced a complete cure, and health has been restored beyond reproach. He died in May next year.

\section{SUMMARY}

1) Eighty - five asthmatics were treated by means of the purified vaccinia lymph.

A few cases were treated with vitamin $B_{2}$ alone or in combination with the former.

A pretty many cases proved a satisfactory effect and have restored to usual health for several months or a year, and, some of them were understood to have been completely cured, while a few considerably improved. In 13 to $21 \%$ of the total cases the treatment seemed to be without benefit.

2) The purified vaccinia lymph is also found effective to the paroxysm of asthmatic tuberculosis and gives to a certain extent a favorable influence on pulmonary tuberculosis.

3) Aged individuals (50-66 years of age) are most readily cured: It is also beneficial to children (2-12 years of age). For young people up to middle ages (14-49 years of age), the method is the most unfavorable.

4) Information as given by patients from time to time, may be summarized as follows :

Although by ordinary medicaments such as ephedrin or adrenalin, only a single attack during the paroxismal period, may be controlled, by the present method, it seems, nearly all attacks in a term, may be averted. If the former is regarded as a momentary sedative, the latter may be said to be an effective measure for a permanent result.

When the treatment was started at an early stage of attack, the aggravations are mostly prevented, but if not commenced until the advanced stage, only a slight effect can be expected.

5) The treatment of patients by the purified vaccinia virus seems to improve the general health. This seems to be due not only to the indirect influence such as the rest resulting from the absence of attacks, but also to some direct action since, the mice inoculated with living purified vaccinia virus show a greater body weight than the animals inoculated with the same heat-killed virus. For example a mouse weighing 8 grams 
inoculated with living vaccinia virus, weighed 12,5 grams in average after 3 weeks, while the same-sized animal, treated by heat-killed virus weighed only 10.3 grams in average in the same period of time.

Furthermore, according to Prof. R. Kinoshita and others, the virus exerts also a beneficial effect on hay-fever.

Briefly, then, the above observations lead to a conclusion that the purified vaccinia lymph is useful as a therapeutic agent for allergic diseases in general.

\title{
REFERENCES
}

1. Yaoi, H.: Combined active immunization against smallpox and typhoid fever (experimental studies). Japan. J. Exp. Med., Vol. 17, No. 3, 295-303, 1939.

2. Yaoi, H., Hirose, S. and Sudzuki, Y.: On the practicability of the combined active immunization against smallpox and typhoid fever (clinical studies). Japan. J. Exp. Med., Vol. 17, No. 3, 305317, 1939.

3. Yaoi, H., Kajiwara, H. and Tomeoka, N.: Combined vaccination by means of the typhoid, cholera vaccines and the purified vaccinia virus (animal experiment). Jikken Igaku, Vol. 24, No. 5, 617-627, 1940.

4. Yaoi, H.: Combined vaccination by means of the typhoid, cholera vaccines and the purified vaccinia virus (practice on man), Jikken Igaku, Vol. 24, No. 12, 1565-1585, 1940.

5. Honma, S. and Yaoi, H.: On the therapeutic effect of the combined vaccination of typhoid vaccine and purified vaccinia virus (Report 1). Nippon Densenbyogakkai Zasshi, Vol. 15, No. 9, 884-885, 1941.

6. Yaoi, H.: Combined vaccination by means of the typhoid, dysentery vaccines and the purified vaccinia virus. Japan. J. Exp. Med., Vol. 19, No, 4, 43-55, 1941.

7. Yaoi, H.: On the treatment of whooping cough, by means of the combined vaccination of $\mathrm{H}$. pertussis vaccine and purified vaccinia lymph. Jikken Igaku, Vol. 26, No. 1, 84-85, 1942.

8. Yaoi, H.: Treatment of whooping cough by combined vaccination of $H$. pertussis vaccine and purified vaccinia lymph (1). Japan. Med. J. Vol. 1, No. 1, 1-4, 1948,

9. Yaoi, H., Arakawa, S. and Nagashima, M.: On the desensitizing action of vitamin $B_{2}$ (Riboflavin) as shown by the anaphylactic shock. Nippon Igaku, No. 3317, 160-163, 1947.

\section{ON THE GROWTH.PROMOTING ACTION OF LIVING VACCINIA VIRUS UPON MICE}

\author{
By Hidetake YAOI \\ National Institute of Health of Japan, Tokyo
}

An .indisputable fact observed while treating bronchial asthma with purified vaccinia lymph is a marked improvement of the general physical conditions, the patients feeling not only insured of the restoration of usual health owing to the riddance of the seizures with paroxysms, but also an apparent increase in vital strength as to enable them to work even harder than before.

The author, herewith, has carried out an experiment to ascertain if the purified vaccinia lymph is of any value in promoting of the growth in mice. Mice weighing $8.0 \mathrm{~g}$ as exactly as possible were selected and fed with $2.0 \mathrm{~g}$ of barley and one $\mathrm{g}$ of 